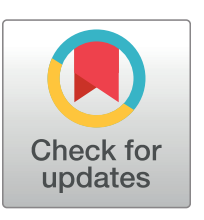

\section{G openaccess}

Citation: Imamura F, Fretts A, Marklund M, Ardisson Korat AV, Yang W-S, Lankinen M, et al. (2018) Fatty acid biomarkers of dairy fat consumption and incidence of type 2 diabetes: $\mathrm{A}$ pooled analysis of prospective cohort studies. PLoS Med 15(10): e1002670. https://doi.org/ 10.1371/journal.pmed. 1002670

Academic Editor: Andrew T Hattersley, University of Exeter, UNITED KINGDOM

Received: April 16, 2018

Accepted: September 7, 2018

Published: October 10, 2018

Copyright: ๑ 2018 Imamura et al. This is an open access article distributed under the terms of the Creative Commons Attribution License, which permits unrestricted use, distribution, and reproduction in any medium, provided the original author and source are credited.

Data Availability Statement: The institutional IRB approvals and data sharing agreements for the participating cohorts allowed us to share cohort results. Individual participant data are owned by individual participating cohorts and are available to researchers consented from participating cohorts. For further queries or requests, please contact force@tufts.edu. Further details are available at the FORCE website: http://force.nutrition.tufts.edu/.

\title{
Fatty acid biomarkers of dairy fat consumption and incidence of type 2 diabetes: A pooled analysis of prospective cohort studies
}

\author{
Fumiaki Imamura $\oplus^{1 *}$, Amanda Fretts ${ }^{2}$, Matti Marklund $\oplus^{3}$, Andres V. Ardisson Korat ${ }^{4}$, \\ Wei-Sin Yang ${ }^{5}$, Maria Lankinen ${ }^{6}$, Waqas Qureshi ${ }^{7}$, Catherine Helmer ${ }^{8}$, Tzu-An Chen ${ }^{9}$, \\ Kerry Wong ${ }^{10}$, Julie K. Bassett ${ }^{10}$, Rachel Murphy ${ }^{11}$, Nathan Tintle ${ }^{12}$, Chaoyu lan $\mathrm{Yu}^{13}$, \\ Ingeborg A. Brouwer $\oplus^{14}$, Kuo-Liong Chien $\varpi^{5}$, Alexis C. Frazier-Wood $\oplus^{9}$, Liana \\ C. del Gobbo ${ }^{15}$, Luc Djoussé ${ }^{16}$, Johanna M. Geleijnse ${ }^{17}$, Graham G. Giles $\oplus^{10,18}$, \\ Janette de Goede $\oplus^{17}$, Vilmundur Gudnason $\oplus^{19}$, William S. Harris $\oplus^{20,21}$, \\ Allison Hodge ${ }^{10,18}$, Frank Hu${ }^{4}$, InterAct Consortium ${ }^{111}$, Albert Koulman $\oplus^{1,22,23,24,25}$, \\ Markku Laakso ${ }^{26}$, Lars Lind ${ }^{27}$, Hung-Ju Lin ${ }^{28}$, Barbara McKnight ${ }^{13}$, Kalina Rajaobelina ${ }^{8}$, \\ Ulf Risérus $\oplus^{3}$, Jennifer G. Robinson ${ }^{29}$, Cécilia Samieri ${ }^{8}$, David S. Siscovick ${ }^{30}$, \\ Sabita S. Soedamah-Muthu $\oplus^{17,31}$, Nona Sotoodehnia ${ }^{2}$, Qi Sun $\oplus^{4}$, Michael Y. Tsai ${ }^{32}$, \\ Matti Uusitupa ${ }^{6}$, Lynne E. Wagenknecht ${ }^{33}$, Nick J. Wareham ${ }^{1}$, Jason $\mathrm{HY} \mathrm{Wu}^{34}$, \\ Renata Micha $\circledast^{35}$, Nita G. Forouhi $\oplus^{1}$, Rozenn N. Lemaitre ${ }^{2}$, Dariush Mozaffarian $\oplus^{35}$, \\ Fatty Acids and Outcomes Research Consortium (FORCE)"
}

1 MRC Epidemiology Unit, University of Cambridge School of Clinical Medicine, Cambridge, United Kingdom, 2 Cardiovascular Health Research Unit, Department of Medicine, University of Washington, Seattle, Washington, United States of America, 3 Department of Public Health and Caring Sciences, Clinical Nutrition and Metabolism, Uppsala University, Sweden, 4 Department of Nutrition and Epidemiology, Harvard T. H. Chan School of Public Health, Boston, Massachusetts, United States of America, 5 Institute of Epidemiology and Preventive Medicine, College of Public Health, National Taiwan University, Taipei City, Taiwan, 6 Institute of Public Health and Clinical Nutrition, University of Eastern Finland, Kuopio, Finland, 7 Section of Cardiovascular Medicine, Department of Internal Medicine, Wake Forest University School of Medicine, Bowman Gray Center, Winston-Salem, North Carolina, United States of America, 8 INSERM, UMR 1219, Bordeaux Population Health Research Center, University of Bordeaux, Bordeaux, France, 9 USDA/ARS Children's Nutrition Research Center, Department of Pediatrics, Baylor College of Medicine, Houston, Texas, United States of America, 10 Cancer Epidemiology and Intelligence Division, Cancer Council Victoria, Melbourne, Australia, 11 Centre of Excellence in Cancer Prevention, School of Population \& Public Health, Faculty of Medicine, The University of British Columbia, Vancouver, Canada, 12 Department of Mathematics and Statistics, Dordt College, Sioux Center, lowa, United States of America, 13 Department of Biostatistics, University of Washington School of Public Health, Seattle, Washington, United States of America, 14 Department of Health Sciences, Faculty of Earth \& Life Sciences, Vrije Universiteit Amsterdam, Amsterdam Public Health Research Institute, Amsterdam, the Netherlands, 15 Department of Medicine, Division of Cardiovascular Medicine, Stanford University School of Medicine, Stanford, California, United States of America, 16 Divisions of Aging, Department of Medicine, Brigham and Women's Hospital and Harvard Medical School, Boston, Massachusetts, United States of America, 17 Division of Human Nutrition, Wageningen University, Wageningen, the Netherlands, 18 Centre for Epidemiology and Biostatistics, The University of Melbourne, Parkville, Australia, 19 Icelandic Heart Association Research Institute, Holtasmári 1, Kópavogur, Iceland, Iceland, 20 Department of Internal Medicine, Sanford School of Medicine, University of South Dakota, Sioux Falls, South Dakota, United States of America, 21 OmegaQuant Analytics LLC, Sioux Falls, South Dakota, United States of America, 22 National Institute for Health Research Biomedical Research Centres Core Nutritional Biomarker Laboratory, University of Cambridge, Addenbrooke's Hospital, Cambridge, United Kingdom, 23 National Institute for Health Research Biomedical Research Centres Core Metabolomics and Lipidomics Laboratory, University of Cambridge, Addenbrooke's Hospital, Cambridge, United Kingdom, 24 Medical Research Council Elsie Widdowson Laboratory, Cambridge, United Kingdom, 25 Institute of Clinical Medicine, Internal Medicine, University of Eastern Finland, Kuopio, Finland,

26 Department of Medicine, Kuopio University Hospital, Kuopio, Finland, 27 Department of Medical Sciences, Uppsala University, Uppsala, Sweden, 28 Department of Internal Medicine, National Taiwan University Hospital, Zhongzheng District, Taipei City, Taiwan, 29 Departments of Epidemiology and Medicine at the University of lowa College of Public Health, lowa City, lowa, United States of America, 30 The New 
Funding: FI, NGF, and NJW were funded by the United Kingdom Medical Research Council Epidemiology Unit core grant (MC_UU_12015/1 and MC_UU_12015/5); NGF, NJW, and AK acknowledge National Institute for Health Research Biomedical Research Centre Cambridge (IS-BRC1215-20014); IAB, by the European Union (FP7 and Horizon2020) and the Dutch Scientific Organisation (ZonMW); CS, by the Fondation Plan Alzheimer; and AVAK, by the National Institute of Health (NIH) training grant (3T32DK007703). The $\mathrm{AOC}$ was funded by the Netherlands Heart Foundation (grant 2000T401), the NIH (NIH/ National Heart, Lung, and Blood Institute [NHLBI], and ODS, grant R01HL- 076200), and Unilever R\&D, Vlaardingen (margarine production and distribution); Age, Gene/Environment Susceptibility Study Reykjavik, by Office of Dietary Supplements, NIH (N01-AG012100), the National Institute of Aging (NIA) Intramural Research Program, Hjartavernd (the Icelandic Heart Association), and the Althingi (the Icelandic Parliament); Chin-Shan Community Cardiovascular Study, by Ministry of Science and Technology and National Taiwan University (MOST 103-2314-B-002 -135 -MY3, NSC 100-2314-B-002 -113 -MY3, NTUH 105S3120, NTUH 106-S3453); Cardiovascular Health Study, by NHLBI (HHSN268201200036C, HHSN268200800007C, N01HC55222, N01HC85079, N01HC85080, N01HC85081, N01HC85082, N01HC85083, N01HC85086, and grants U01HL080295 and U01HL130114), with additional contribution from the National Institute of Neurological Disorders and Stroke (NINDS) and NIA (R01AG023629; Framingham Heart Study, NHLBI in collaboration with Boston University (N01-HC-25195); Health Professionals Follow-up Study, by NIH (UM1 CA167552, R01 HL35464, AA11181, HL35464, CA55075, HL60712, and P30 DK46200); Insulin Resistance Atherosclerosis Study, by NIH (M01-RR-43) and NHLBI (U01-HL47892, U01-HL-47902, DK-29867, R01-58329, and DK-079888; InterAct, by the EU FP6 programme (LSHM_CT_2006_037197); Melbourne Collaborative Cohort Study, by VicHealth and Cancer Council Victoria and by Australia's National Health and Medical Research Council (209057, 251553, and 126403); Multiethnic Study of Atherosclerosis, by NHLBI (HHSN268201500003I, N01-HC-95159, N01-HC95160, N01-HC-95161, N01-HC-95162, N01-HC95163, N01-HC-95164, N01-HC-95165, N01-HC95166, N01-HC-95167, N01-HC-95168 and N01HC-95169) and NCRR (UL1-TR-000040 and UL1TR-001079); Metabolic Syndrome in Men Study, by the European Union, the Academy of Finland, and the Juselius Foundation; Nurses' Health Study,
York Academy of Medicine, New York, New York, United States of America, 31 Center of Research on Psychology in Somatic Diseases, Department of Medical and Clinical Psychology, Tilburg University, Tilburg, the Netherlands, 32 Department of Laboratory Medicine and Pathology, University of Minnesota, Minneapolis, Minnesota, United States of America, 33 Public Health Sciences, Wake Forest School of Medicine, Winston-Salem, North Carolina, United States of America, 34 The George Institute for Global Health and the Faculty of Medicine, University of New South Wales, Sydney, Australia, 35 Friedman School of Nutrition Science and Policy, Tufts University, Boston, Massachusetts, United States of America

I Membership of the InterAct Consortium and the Fatty Acids and Outcomes Research Consortium (FORCE) is linked to in the Acknowledgements.

* fumiaki.imamura@mrc-epid.cam.ac.uk

\section{Abstract}

\section{Background}

We aimed to investigate prospective associations of circulating or adipose tissue odd-chain fatty acids $15: 0$ and 17:0 and trans-palmitoleic acid, $t 16: 1 \mathrm{n}-7$, as potential biomarkers of dairy fat intake, with incident type 2 diabetes (T2D).

\section{Methods and findings}

Sixteen prospective cohorts from 12 countries ( 7 from the United States, 7 from Europe, 1 from Australia, 1 from Taiwan) performed new harmonised individual-level analysis for the prospective associations according to a standardised plan. In total, 63,682 participants with a broad range of baseline ages and BMls and 15,180 incident cases of T2D over the average of 9 years of follow-up were evaluated. Study-specific results were pooled using inverse-variance-weighted meta-analysis. Prespecified interactions by age, sex, BMI, and race/ethnicity were explored in each cohort and were meta-analysed. Potential heterogeneity by cohort-specific characteristics (regions, lipid compartments used for fatty acid assays) was assessed with metaregression. After adjustment for potential confounders, including measures of adiposity (BMI, waist circumference) and lipogenesis (levels of palmitate, triglycerides), higher levels of 15:0, 17:0, and $t 16: 1 \mathrm{n}-7$ were associated with lower incidence of T2D. In the most adjusted model, the hazard ratio $(95 \% \mathrm{Cl})$ for incident T2D per cohortspecific 10th to 90 th percentile range of $15: 0$ was $0.80(0.73-0.87)$; of $17: 0,0.65(0.59-$ $0.72)$; of $t 16: 1 \mathrm{n} 7,0.82(0.70-0.96)$; and of their sum, $0.71(0.63-0.79)$. In exploratory analyses, similar associations for 15:0,17:0, and the sum of all three fatty acids were present in both genders but stronger in women than in men $\left(p_{\text {interaction }}<0.001\right)$. Whereas studying associations with biomarkers has several advantages, as limitations, the biomarkers do not distinguish between different food sources of dairy fat (e.g., cheese, yogurt, milk), and residual confounding by unmeasured or imprecisely measured confounders may exist.

\section{Conclusions}

In a large meta-analysis that pooled the findings from 16 prospective cohort studies, higher levels of 15:0, 17:0, and $t 16: 1 \mathrm{n}-7$ were associated with a lower risk of T2D. 
by NIH (CA186107, CA87969, CA49449, HL34594, HL35464, CA167552, HL60712, and HL088521); Prospective Investigation of the Vascularture in Uppsala Seniors, by Uppsala University Hospital and the Swedish Research Council for Health, Working Life and Welfare; Tree City Study, by the Fondation pour la Recherche Medicale, the Caisse Nationale Maladie des Travailleurs Salaries, Direction Generale de la Sante, MGEN, Institut de la Longevite, Conseils Regionaux d'Aquitaine et Bourgogne, Fondation de France, Ministry of Research-Institut National de la Sante and de la Recherche Medicale Programme Cohortes, Agence Nationale de la Recherche (COGINUT ANR-06PNRA-005), Fondation Plan Alzheimer (FCS 20092012), and the Caisse Nationale pour la Solidarite et l'Autonomie, under a partnership agreement between the Institut National de la Sante et de la Recherche Medicale, the University Bordeaux 2 Victor Segalen; Uppsala Longitudinal Studies of Adult Men 50 and 70, by the Swedish Research Council for Health, Working Life and Welfare, Uppsala City Council, and Swedish Research Council; Women's Health Initiative, by the NHLBI, $\mathrm{NIH}$, U.S. Department of Health and Human Services (HHSN268201600018C, HHSN268201600001C, HHSN268201600002C, HHSN268201600003C, and HHSN268201600004C). The funders had no role in study design, data collection and analysis, decision to publish, or preparation of the manuscript.

Competing interests: I have read the journal's policy and the authors of this manuscript have the following competing interests: JHYW and RM report research support from Unilever for other projects of the FORCE on other fatty acid biomarkers. RM reports personal fees from the World Bank and Bunge outside the submitted work. IAB reported involvement in a research project partly funded by Unilever. JMG and JdG received funding from Unilever for epidemiological studies of dietary and circulating fatty acids and cardiometabolic disease and for research on assessment of fatty acids. LCdG reported receiving ad hoc consulting fees from the Life Sciences Research Organization. $\mathrm{CH}$ reported receiving fees for a conference from Novartis. NGF is an invited member (unpaid) of ILSI-Europe Qualitative Fat Intake Task Force Expert Group on update on health effects of different saturated fats. DM reports research funding from the $\mathrm{NIH}$ and the Gates Foundation; personal fees from GOED, DSM, Nutrition Impact, Pollock Communications, Bunge, Indigo Agriculture, Amarin, Acasti Pharma, and America's Test Kitchen; scientific advisory board, Omada Health, Elysium Health, and DayTwo; and chapter royalties from UpToDate; all outside the

\section{Author summary}

\section{Why was this study done?}

- Effects of dairy fat on type 2 diabetes (T2D) are not well established. While dairy fat contains palmitic acid that could increase risk of T2D, it also contains several other types of fatty acids and further reflects specific foods, such as cheese or yogurt, that could reduce risk.

- Most prior studies of dairy foods and T2D have relied on self-reported dietary questionnaires, which may have errors or bias in memory as well as challenges in assessing less apparent sources of dairy fat such as in creams, sauces, cheeses, and cooking fats in mixed meals and prepared foods.

- Circulating and tissue biomarker concentrations of odd-chain saturated fats (15:0, 17:0) and natural ruminant trans-fats (trans-16:1n7) at least partly reflect dairy fat consumption, help capture multiple dietary sources without relying on memory or subjective reporting, and reflect a complementary approach to investigate associations with T2D.

- A consortium strategy combining all available studies maximises statistical power and generalizability, allows standardised analytical approaches and methods including of key population subgroups, and minimises potential for publication bias.

\section{What did the researchers do and find?}

- We conducted a consortium project to pool new participant-level analyses of 16 cohort studies as part of the Fatty Acids and Outcomes Research Consortium (FORCE), including a total of 63,682 adults free of T2D at baseline, among whom 15,158 developed incident T2D over up to 20 years of follow-up.

- Participating studies conducted standardised analysis of the prospective associations between fatty acid biomarkers (15:0, 17:0, trans-16:1n7, and their sum) and the risk of developing T2D.

- Pooling all studies, each of the biomarkers and their sum were associated with lower risk of developing T2D, independently of major risk factors for T2D, including age, sex, race/ethnicity, socioeconomic status, physical activity, and obesity.

- For example, for the sum of these biomarkers, participants with higher levels experienced $29 \%$ (95\% CI 21\% to 37\%) lower risk of T2D than adults with lower levels, comparing between the midpoints of the top fifth and the bottom fifth of concentrations.

\section{What do these findings mean?}

- Higher circulating and tissue concentrations of odd-chain saturated fats and a natural ruminant trans-fat are associated with lower risk of T2D.

- While these biomarkers are known to reflect dairy fat consumption, their levels could also be influenced by other unknown factors. The findings support the need for 
submitted work. Patents US8889739 and US9987243 to Tufts University (unlicensed), listing DM as a co-inventor, for use of trans-palmitoleic acid to prevent and treat insulin resistance, type 2 diabetes, and related conditions, as well as reduce metabolic risk factors. SSSM reported receiving an international award and unrestricted grants for meta-analysis work on dairy foods and cardiometabolic diseases from Global and Dutch Dairy Associations. Other authors do not have any conflict of interest to declare. The lead author affirms that the manuscript is an honest, accurate, and transparent account of the study being reported; that no important aspects of the study have been omitted; and that any discrepancies from the study as planned (and, if relevant, registered) have been explained.

Abbreviations: AGESR, Age, Genes, Environment Susceptibility Study (Reykjavik); AOC, Alpha Omega Cohort; CCCC, Chin-Shan Community Cardiovascular Cohort Study; CHS, Cardiovascular Health Study; FHS, Framingham Heart Study; FORCE, Fatty Acids and Outcomes Research Consortium; HPFS, Health Professionals' Follow-up Study; MCCS, Melbourne Collaborative Cohort Study; MESA, Multi-Ethnic Study of Atherosclerosis; METSIM, Metabolic Syndrome in Men Study; NHS, Nurses' Health Study; PIVUS, Prospective Investigation of the Vasculature in Uppsala Seniors; RR, relative risk; T2D, type 2 diabetes mellitus; Three C, Three City Study; ULSAM, Uppsala Longitudinal Study of Adult Men; WHIMS, Women's Health Initiative Memory Study. investigation of determinants of levels of these fatty acids as well as health effects of dairy fat in interventional studies.

- Despite the several advantages of evaluating fatty acid biomarkers, the results cannot distinguish between different types of dairy foods (e.g., milk, cheese, yogurt, others), which could have differential effects.

- The findings provide the strongest evidence to date for relationships of these fatty acid biomarkers with T2D, informing the potential health effects and corresponding dietary recommendations for consumption of selected dairy products.

\section{Introduction}

Regular consumption of dairy products is widely recommended in national and international guidelines as a major source of calcium and other minerals and vitamins as well as in lowincome countries as a source of calories and protein. At least in high-income nations, fatreduced dairy products are further recommended, rather than whole-fat products, with the aim of limiting calories and saturated fat [1]. However, these latter recommendations are primarily based on nutrient profiles of low-fat and whole-fat dairy products rather than empirical evidence on clinical effects of dairy fat from prospective observational studies or trials [2-8]. In clinical trials, consuming low-fat or free-fat dairy products does not consistently improve intermediate risk factors compared to consuming whole-fat or overall dairy products [2-4]. In observational studies, total dairy consumption has not been associated with cardiovascular diseases, without consistent distinction based on dairy fat content. Regardless of fat content, total dairy consumption has been associated with lower incidence of type 2 diabetes (T2D) [8], whereas evidence is inconsistent for different types of dairy foods such as milk, yogurt, and cheese.

Studies assessing dairy consumption using self-reported dietary questionnaires may be partly limited by misclassification or bias in reporting [9]. In addition, the common use of dairy products such as butter, milk, cheese, and cream in cooking, in mixed dishes (e.g., pizza), and bakery products (e.g., cakes) may substantially impede an accurate assessment of exposure to dairy fat. To reduce these limitations, measured biomarkers correlated with dairy fat consumption can be used, including circulating and adipose proportions of pentadecanoic acid (15-carbon saturated fatty acid, 15:0), heptadecanoic acid (17:0), and trans-palmitoleic acid $(t 16: \ln 7)$ [10-20]. Levels of these biomarkers correlate with self-reported consumption of total dairy, high-fat dairy, and dairy fat ( $r=0.4$ to 0.7 ) based on 24-hour recalls or 7-day food records [16-18]; are significantly increased in response to dairy consumption or decreased in replacing high-fat dairy with low-fat dairy in trials [19,20]; and are correlated with each other even though they represent two distinct fatty acid classes (the odd-chain saturated fats 15:0 and 17:0; the natural ruminant trans-fat $t 16: 1 \mathrm{n} 7$ ) with divergent chemical structures and metabolism.

To date, several individual cohorts have published on associations of the odd-chain saturated fatty acids only [21] or odd-chain fatty acids and $t 16: 1 \mathrm{n} 7$ together $[13,14,22]$ with incidence of T2D. However, potential for publication bias cannot be excluded; individual studies may be underpowered to detect potential differences in associations by sex or other characteristics [8]. To address these limitations and provide new evidence on relationships between 
these biomarkers and T2D, we conducted a pooling project to test the hypothesis that higher concentrations of 15:0, 17:0, and t16:1n7 would be associated with lower incident T2D, evaluating adults free from T2D in prospective cohorts participating in the Fatty Acids and Outcomes Research Consortium (FORCE).

\section{Methods}

\section{Cohorts and study variables}

FORCE was formed within the framework of the Cohorts for Heart and Aging Research in Genomic Epidemiology consortium fatty acid working group to focus on relationships between fatty acid biomarkers and health outcomes (http://force.nutrition.tufts.edu/about) $[23,24]$. FORCE cohorts were identified through expert contacts with existing large cohorts and publications, with updating over time when new cohort publications were identified. For the current investigation, we included 16 prospective studies (cohorts, nested case-control studies, or nested case-cohort studies) that met the following inclusion criteria and agreed to participate: adult aged 18 years or older free from diabetes at the time of fatty acid assessment; circulating or adipose 15:0, 17:0, or t16:1n7; and follow-up for incident T2D (S1 Text). Other cohorts participating in FORCE $[23,24]$ did not contribute to this study because data on these fatty acids and/or incident T2D were not available. All cohorts obtained institutional review board approval and informed consents from participants. Authors FI and AF have full access to the data that are available upon request to the central committee of FORCE.

A standardised analysis protocol (S2 Text) was developed and was provided to each participating cohort. It included inclusion criteria (adults aged 18 years or older, not with diabetes, and with data on fatty acids and incident T2D), exposures, covariates, effect modifiers, outcomes, and longitudinal analyses. Following this harmonised protocol, each cohort performed new analysis of individual-level data. Study-specific results were entered to a standardised electronic form and compiled centrally; the results were then pooled in meta-analysis [25].

Details of participating cohorts, study participants, fatty acid assessment, ascertainment of incident T2D, and relevant citations are presented in S1 Text; fatty acid concentrations were assessed with gas chromatography in each cohort in one or more lipid compartments, including erythrocyte phospholipids, plasma phospholipids, plasma cholesteryl esters, plasma triglycerides, total plasma, or adipose tissue. Fatty acid concentrations in each cohort were expressed as a percent of total fatty acids in each lipid fraction. In extended analysis of prior work [22], within-person correlations of phospholipid fatty acids were moderate over 6 and 13 years $(n=607)$ ( $r=0.64$ and 0.46 for 15:0, respectively; 0.66 and 0.47 for 17:0; and 0.59 and 0.45 for $t 16: 1 \mathrm{n} 7)$, consistent with other biometric risk factors such as blood pressure [26].

In most cohorts, incident T2D was ascertained based on one or more criteria (S1 Text), including fasting glucose $\geq 126 \mathrm{mg} / \mathrm{dL}(7.0 \mathrm{mmol} / \mathrm{L}) ; 2$-hour post oral glucose tolerance test glucose $\geq 200 \mathrm{mg} / \mathrm{dL}$ (11.1 mmol/L); new use of insulin or oral hypoglycaemic medication assessed by participant reports, medication inventories, or registries (S1 Text); and fasting or nonfasting $\mathrm{HbA} 1 \mathrm{C}$ concentration $\geq 6.5 \%$. In the Melbourne Collaborative Cohort Study (MCCS) [27] and the Alpha Omega Cohort (AOC) [28], incident T2D was defined by selfreported physician diagnosis, use of antidiabetic medication, or both. InterAct defined incident T2D by adjudicating self-reported diagnosis of T2D or data linkage to disease registry [21]. In studies with time-to-event data, follow-up time was calculated from baseline (time of fatty acid measurement) to date of development of incident T2D, death from any cause, loss to follow-up, or censoring at end of follow-up-whichever came first. 


\section{Statistical analysis in individual studies}

Statistical analyses were prespecified to describe population characteristics and conduct prospective analyses of associations of the fatty acid biomarkers and incident T2D. The primary exposure variables were 15:0, 17:0, t16:1n7, and their sum (or if only two were available, the sum of the two). The sum was considered a biomarker of dairy fat intake, given the available evidence that these each of these fatty acids at least partly reflects dairy fat intakes [11-20] and that these fatty acids are mutually intercorrelated [12-16,22]. Pearson correlation coefficients were calculated between these fatty acids in each study and between fractions in different lipid compartments when available in the same cohort.

For prospective associations, Cox proportional hazard regression models were fitted to data from cohort or nested case-cohort studies. In the MCCS [27] without detailed time-to-event data for participants, logistic regression was used. The fatty acids were evaluated as a continuous linear variable in units of the study-specific 10th to 90th percentile range and, in a separate model, as a dummy categorical variable (quintile categories).

Covariates in all multivariable-adjusted analyses were prespecified. The primary model included age, sex, field site, race, education, occupation, physical activity, smoking, alcohol use, prevalent hypertension (treated or self-reported), prevalent dyslipidaemia (treated or selfreported), prevalent coronary heart disease, and self-reported health status. We obtained measures of association from two additional models: one further adjusting for adiposity measures (BMI and waist circumference) and the other further adjusting for circulating concentrations of triglycerides and palmitate (16:0), markers of hepatic de novo lipogenesis. Study-specific approaches were allowed for modelling some covariates (e.g., numbers of education categories, imputation for missing covariates), depending on availability and prior established cohort-specific approaches, to minimise confounding bias within each cohort [25]. Using the multivariable-adjusted model including adiposity measures, we obtained study-specific measures of effect modification by age, sex, BMI, and race/ethnicity (indicator categories with white race as the reference group) by evaluating the coefficient of a crossproduct term between each fatty acid variable and each of the prespecified factors.

\section{Meta-analysis}

Study-specific regression coefficients and measures of precision (standard errors) from each of continuous and categorical terms were pooled with an inverse-variance-weighted meta-analysis to estimate summary relative risks (RRs) per the 10th to 90th percentile range and quintile categories. Between-study heterogeneity was expressed as I-squared [29]. Odds ratios estimated in a study without information on time to event were considered to approximate RRs, and RRs were assumed to represent hazard ratios as well. Four cohorts assessed fatty acids in two lipid compartments: the Prospective Investigation of the Vasculature in Uppsala Seniors (PIVUS) and the AOC evaluated plasma cholesteryl esters and plasma phospholipid fatty acids, and the Nurses' Health Study (NHS) and the Health Professionals' Follow-up Study (HPFS) evaluated total plasma and red blood cell phospholipid fatty acids. In the primary meta-analysis, not to double-count estimates from these cohorts, we used estimates of phospholipid fatty acids that were likely to reflect a longer-term exposure than the other compartments [30]. Estimates from separate fractions were obtained separately as stratified analysis by lipid fractions.

Cohort-specific coefficients of crossproduct terms were pooled by inverse-varianceweighted meta-analysis to test potential interactions. Because analyses of potential interactions by age, sex, race/ethnicity, or BMI were exploratory, we corrected for multiple testing with two-tailed alpha $=0.0031$ (0.05; 4 fatty acid variables; 4 potential effect modifiers). Because 
interactions by sex were significant, we post hoc estimated sex-specific RRs by obtaining relevant statistics from each cohort. We also conducted metaregression and stratified meta-analyses to examine whether associations varied by study-specific characteristics, including lipid compartment, region (the United States, Europe/Australia, Asia), mean prevalence of dyslipidaemia, and numbers of fatty acids assessed. Meta-analyses were performed using Stata software version 14.2 (Stata Corp., College Station, Texas) with alpha $=0.05$ unless otherwise specified.

\section{Results}

The 16 prospective studies ( 7 in the US, 7 in Europe, 1 in Australia, 1 in Taiwan) included 63,682 participants without known diabetes at baseline, among whom 15,180 incident T2D cases were identified during an average 9 years of follow-up (Table 1). All studies followed middle-aged or older adults with baseline mean age in each cohort ranging from 49 to 76 years. Average BMIs ranged from 25.0 to $28.4 \mathrm{~kg} / \mathrm{m}^{2}$ except for Taiwan with an average BMI of $23.3 \mathrm{~kg} / \mathrm{m}^{2}$. Most studies included predominantly white participants, although meaningful numbers of nonwhites were included in the Cardiovascular Health Study (CHS; 11.0\% nonwhite), the Multi-Ethnic Study of Atherosclerosis (MESA; 71.6\% nonwhite), the Women's Health Initiative Memory Study (WHIMS; 11.6\% nonwhite), and the Taiwanese study (100\% Asian).

Relative concentrations of $15: 0,17: 0$, and $t 16: 1 \mathrm{n} 7$ were generally low $(0.1 \%$ to $0.5 \mathrm{~mol} \%$ of total fatty acids), as previously described (Fig 1) [13,14,21,22]. Correlations between 15:0, 17:0, and $t 16: 1 \mathrm{n} 7$ ranged from 0.3 to 0.8 , with the exception of $r=0.0$ in the Insulin Resistance Atherosclerosis Study and WHIMS (S1 Table). Correlations of each of the fatty acids between two lipid fractions (e.g., phospholipids and total plasma; phospholipids and cholesteryl esters) were also moderate to strong ( $r=0.39$ to 0.75$)$ (S2 Table).

In meta-analysis of 15:0 (16 cohorts, 59,701 participants, 14,658 cases), higher 15:0 levels were associated with $26 \%$ lower risk of $\mathrm{T} 2 \mathrm{D}$ (per 10th to 90th percentile range, pooled $\mathrm{RR}=0.74$ [95\% CI 0.68-0.80]) adjusted for demographic, clinical, socioeconomic, and lifestyle variables ( $\mathrm{S} 1 \mathrm{Fig}) ; 20 \%$ lower risk $(\mathrm{RR}=0.80$ [95\% CI 0.74-0.87]) when further adjusted for adiposity measures (Fig 2); and $20 \%$ lower risk ( $\mathrm{RR}=0.80$ [95\% CI 0.73-0.87]) when further adjusted for biomarker concentrations of palmitic acid and triglycerides (S2 Fig). Inverse associations were also observed for 17:0 (13 cohorts, 50,579 participants, 13,720 cases), t16:1n7 (8 studies, 18,901 participants, 1,636 cases), and the sum of dairy biomarker fatty acids (15 studies, 53,550 participants, 14,175 cases). In post hoc sensitivity analyses excluding the study with the largest weight (InterAct or WHIMS, Fig 2), results were not substantially altered: RR per 10th to 90th percentile range ( $95 \% \mathrm{CI}$ ) for 15:0, 0.75 (95\% CI 0.62-0.92); for 17:0, 0.73 (95\% CI 0.55-0.96); for t16:1n7, 0.84 (95\% CI 0.72-0.98); and for their sum, 0.75 (95\% CI 0.57-0.99).

Results were similar evaluating risk across quintile groups of each fatty acid including in each multivariable model (Fig 3). Comparing the top to the bottom quintile of fatty acid levels in the fully adjusted model, RRs (95\% CI) were $0.63(0.52-0.76)$ for 15:0, $0.64(0.47-0.87)$ for $17: 0,0.83(0.62-1.11)$ for $t 16: 1 \mathrm{n} 7$, and $0.65(0.51-0.83)$ for their sum. Moderate to high heterogeneity was evident ( $\mathrm{I}^{2}$ ranging from $60 \%$ to $90 \%$ ) (Fig 2, S1 Fig, S3 Fig), except for $t 16: 1 n 7\left(\mathrm{I}^{2}\right.$ $0 \%$ to $7.7 \%$ ). Results of post hoc analysis estimating random effects were similar (S3 Table).

In exploratory analyses, the inverse association with $\mathrm{T} 2 \mathrm{D}$ was stronger in women than in men for 15:0 ( $\left.p_{\text {interaction }}=0.0003\right), 17: 0\left(p_{\text {interaction }}=0.003\right)$, and the sum of the fatty acids $\left(p_{\text {interaction }}=0.0003\right)$, with women experiencing a $20 \%$ to $27 \%$ lower risk than men (S3 Table). For example, RR ( $95 \% \mathrm{CI})$ per 10th to 90th percentile range for 15:0 was $0.76(0.69-0.84)$ for women and $0.93(0.85-1.01)$ for men. Metaregression did not identify any other significant 
Table 1. Baseline characteristics of 16 studies of the pooling analysis of fatty acid biomarkers (15:0, 17:0, trans-16:1n7) and incident T2D ${ }^{a}$.

\begin{tabular}{|c|c|c|c|c|c|c|c|c|c|c|}
\hline Study & Country & $\begin{array}{l}\text { Study } \\
\text { design }\end{array}$ & $\begin{array}{l}\text { Baseline } \\
\text { year }\end{array}$ & $\begin{array}{l}\text { Follow-up } \\
\text { years, median }\end{array}$ & $\begin{array}{l}N \text { adults ( } N \\
\text { cases) }\end{array}$ & $\begin{array}{l}\text { Age, mean } \\
\text { years }\end{array}$ & $\begin{array}{l}\text { Sex, \% } \\
\text { women }\end{array}$ & $\begin{array}{l}\text { BMI, mean } \\
\left(\mathrm{kg} / \mathrm{m}^{2}\right)\end{array}$ & $\begin{array}{l}\text { Biomarker } \\
\text { compartment }\end{array}$ & $\begin{array}{l}N \text { fatty acids } \\
\text { assessed }\end{array}$ \\
\hline CHS & United States & Cohort & 1992 & 10.6 & $3,179(284)$ & 75.1 & 61.5 & 26.4 & PL & 45 \\
\hline MESA & United States & Cohort & 2000-2002 & 9.3 & $2,252(309)$ & 61.0 & 53.9 & 27.6 & PL & 27 \\
\hline IRAS & United States & Cohort & $1992-1997$ & 5.3 & $719(146)$ & 55.1 & 55.8 & 28.4 & Total plasma & 34 \\
\hline FHS & United States & Cohort & 2005-2008 & 5.8 & $2,209(98)$ & 64.4 & 57.2 & 27.8 & RBC PL & 33 \\
\hline WHIMS & United States & Cohort & 1996 & 11.0 & $6,510(502)$ & 70.1 & 100 & 28.1 & RBC PL & 22 \\
\hline NHS & United States & Cohort & 1990 & 16.9 & $1,760(177)$ & 60.4 & 100 & 25.3 & $\begin{array}{l}\text { RBC PL, total } \\
\text { plasma }\end{array}$ & 37 \\
\hline HPFS & United States & Cohort & 1994 & 11.1 & $1,519(112)$ & 64.1 & 0 & 25.8 & $\begin{array}{l}\text { RBC PL, total } \\
\text { plasma }\end{array}$ & 37 \\
\hline Inter Act $^{\mathrm{b}}$ & $\begin{array}{l}\text { Eight European } \\
\text { countries }\end{array}$ & $\begin{array}{l}\text { Case } \\
\text { cohort }\end{array}$ & 1993-1997 & 12.3 & $\begin{array}{l}27,296 \\
(12,132)\end{array}$ & 52.3 & 62.3 & 26.0 & PL & 37 \\
\hline AGESR & Iceland & Cohort & $2002-2006$ & 5.2 & $753(28)$ & 75.5 & 59.5 & 27.0 & PL & 41 \\
\hline Three C & France & Cohort & $1999-2000$ & 8.0 & $565(39)$ & 76.0 & 64.3 & 25.0 & RBC PL & 35 \\
\hline $\mathrm{AOC}$ & The Netherlands & Cohort & $2002-2006$ & 2.5 & $760(37)^{\mathrm{c}}$ & 68.9 & 20.4 & 27.4 & RBC PL, CE & 38 \\
\hline ULSAM & Sweden & Cohort & $1970-1973$ & 21.4 & $2,009(396)$ & 54.4 & 0 & 25.2 & Adipose tissue & 17 \\
\hline PIVUS & Sweden & Cohort & 2001-2004 & 10.0 & $879(67)$ & 72.5 & 51.0 & 26.7 & PL, CE & 16 \\
\hline METSIM & Finland & Cohort & 2006-2010 & 5.5 & $1,302(71)$ & 57.3 & 0 & 26.4 & PL & 22 \\
\hline MCCS & Australia & $\begin{array}{l}\text { Case } \\
\text { cohort }\end{array}$ & 1990-1994 & 4.0 & $6,151(490)$ & 56.3 & 53.9 & 27.0 & PL & 53 \\
\hline CCCC & Taiwan & Cohort & 1992-1993 & 6.0 & $1,838(128)$ & 58.7 & 40.0 & 23.2 & Total plasma & 29 \\
\hline
\end{tabular}

${ }^{\mathrm{a}}$ Characteristics at the time of fatty acid biomarker measurement.

${ }^{\mathrm{b}}$ Upon a decision within the cohort, InterAct provided pooled estimates from eight European countries: France, Spain, the United Kingdom, Sweden, Germany, Italy,

Denmark, and the Netherlands.

${ }^{c}$ The AOC evaluated 1,741 participants (201 incident cases) with CE measures that were analysed in secondary analyses.

Abbreviations: AGESR, Age, Genes, Environment Susceptibility Study (Reykjavik); AOC, Alpha Omega Cohort; CCCC, Chin-Shan Community Cardiovascular Cohort Study; CE, cholesteryl ester; CHS, Cardiovascular Health Study; FHS, Framingham Heart Study; HPFS, Health Professionals' Follow-up Study; MCCS, Melbourne Collaborative Cohort Study; MESA, Multi-Ethnic Study of Atherosclerosis; METSIM, Metabolic Syndrome in Men Study; NHS, Nurses' Health Study; PIVUS,

Prospective Investigation of the Vasculature in Uppsala Seniors; PL, phospholipid; RBC, red blood cell; Three C, Three City Study; ULSAM, Uppsala Longitudinal Study of Adult Men; WHIMS, Women's Health Initiative Memory Study.

sources of heterogeneity ( $p_{\text {interaction }}>0.1$ each), including by geographic region, measured lipid compartment, prevalence of dyslipidaemia, or the number of fatty acids assessed (S3 Table).

\section{Discussion}

This harmonised pooling project of participant-level data among 16 prospective cohort studies provides, to our knowledge, the most comprehensive evidence for associations of biomarker levels of 15:0, 17:0, and $t 16: 1 \mathrm{n} 7$ with risk of T2D. Comparing the top to the bottom quintile of participants in each cohort, we found that higher levels of the sum of these fatty acids were associated with approximately $30 \%$ lower risk of developing T2D. This relationship remained significant after adjustment for demographic characteristics, socioeconomic status, lifestyle factors, medical history, adiposity measures, and biomarkers of de novo lipogenesis.

Measured circulating and tissue levels of 15:0, 17:0, and $t 16: 1 \mathrm{n} 7$ are free from bias in relation to memory or reporting. Compared with estimated dairy fat intake from self-reported questionnaires, direct measurement also facilitates assessment of exposure to numerous 'hidden' sources of dairy fat in the food supply, e.g., as found in many dishes that include varying 


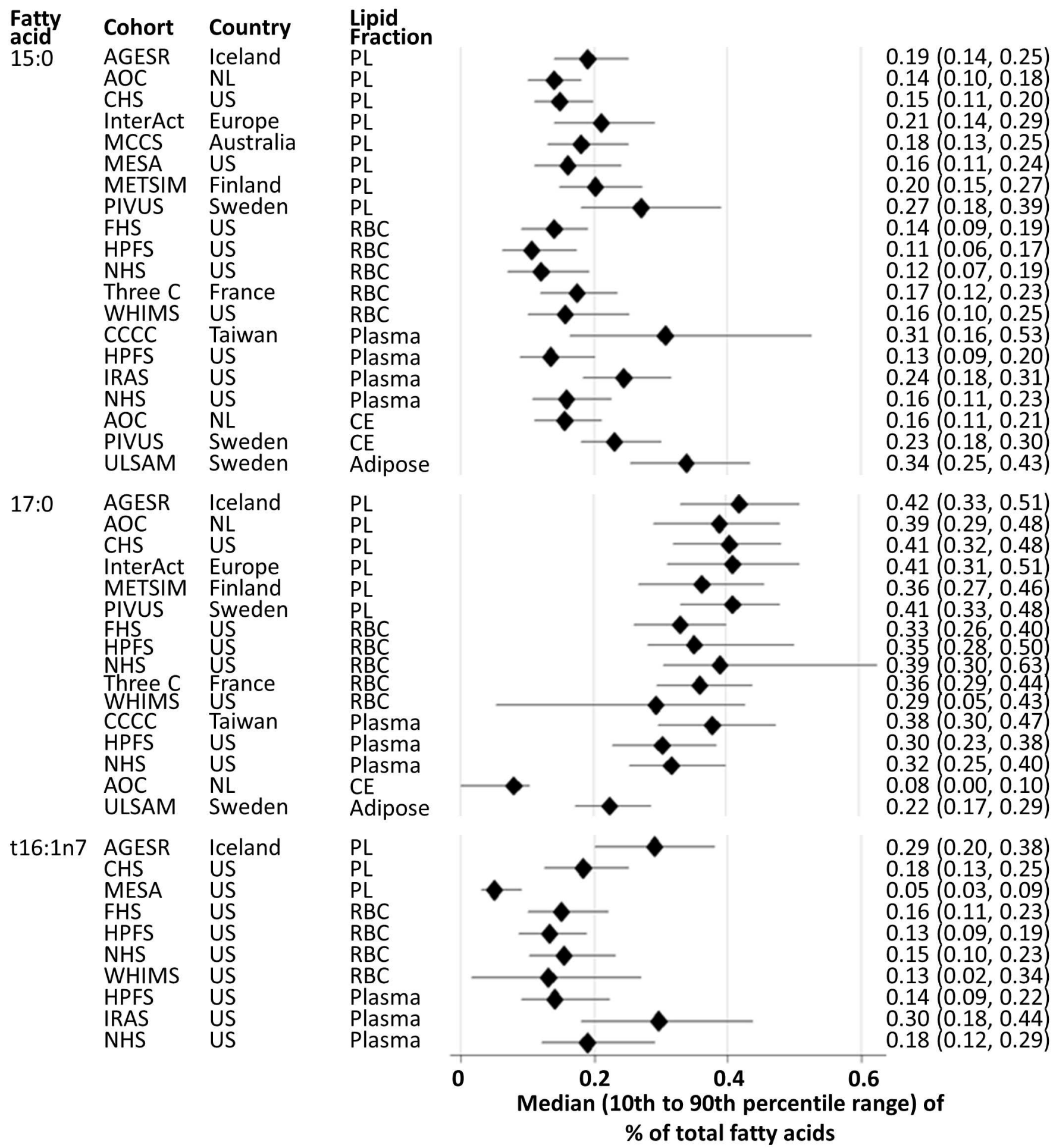

Fig 1. Proportions of fatty acid biomarkers for dairy fat consumption. Plots represent median (diamond) and ranges of the 10th to 90 th percentiles (horizontal bar). See Table 1 for the abbreviations of cohorts. CE, cholesteryl ester; NL, the Netherlands; PL, phospholipid; RBC, red blood cell; t16:1n7, trans16:1 n-7; US, United States.

https://doi.org/10.1371/journal.pmed.1002670.g001 


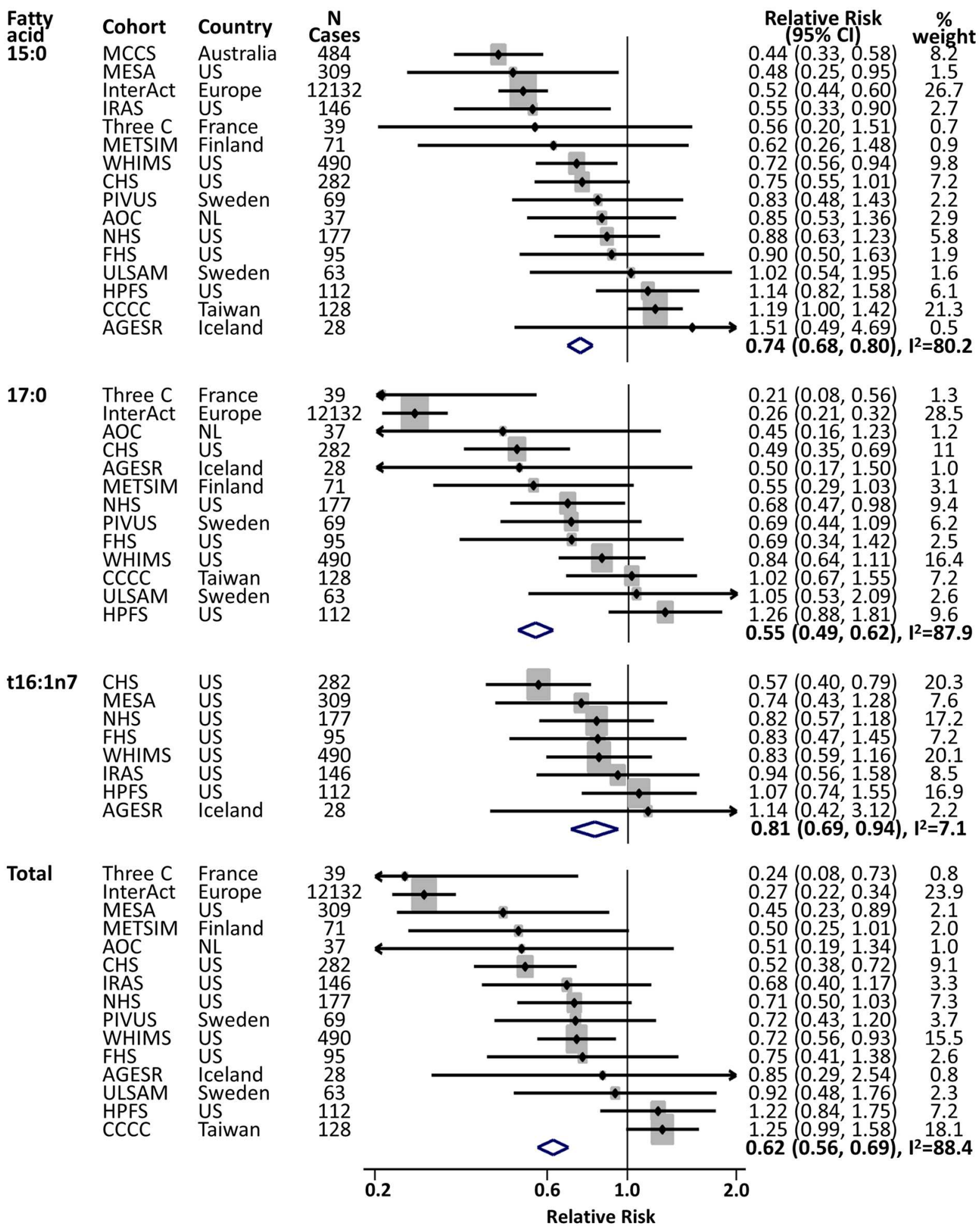

Fig 2. Prospective associations of fatty acid biomarkers for dairy fat consumption with the risk of developing T2D. RR and 95\% CIs per cohort-specific range from the 10th to 90th percentiles are presented: dots from individual studies and diamonds as summary estimates pooled by inverse-variance-weighted meta-analysis. The sizes of the grey shaded areas represent relative contributions of each cohort to that summary estimate. Cohort-specific association was assessed in multivariable models in each cohort adjusting for sex, age, field site (if appropriate), race, socioeconomic status (education, occupation), smoking status, physical activity, alcohol consumption, family history of diabetes,

dyslipidaemia, hypertension, menopausal status (only for women), prevalent coronary heart disease, BMI, and waist circumference. Models 
without the adiposity measures and models including palmitate (16:0) and triglycerides did not alter the results materially (S1 Fig). See Table 1 for the abbreviations of cohorts. NL, the Netherlands; RR, relative risk; T2D, type 2 diabetes mellitus; US, United States.

https://doi.org/10.1371/journal.pmed.1002670.g002

amounts of sauces, creams, and butter, milk, or cheese as mixed or prepared meals. Odd-chain saturated fats can be found in other foods, such as meat or fish [31,32], and their blood levels are measurable among self-reported vegans [10]. However, levels among vegans are significantly lower than among lacto-ovo vegetarians, supporting a sensitivity of the biomarkers to dairy fat consumption [10]. Several additional lines of evidence support a role of these fatty acids as biomarkers reflecting consumption of dairy fat and high-fat dairy products. First, among different food groups, correlations of these fatty acid biomarkers are strongest with dairy foods and dairy fat $[12,15,16]$. Such correlations are generally low to modest $(r=0.1$ to 0.5 ) in studies using food-frequency questionnaires (which may miss many 'hidden' sources of dairy fat) $[12,13,21]$ but much stronger $(r=0.4$ to 0.7$)$ in studies evaluating 24 -hour recalls or 7-day food records, which much more completely capture the types and details of specific foods consumed [16-18]. Second, in controlled interventions, levels of these fatty acids are significantly increased or decreased in response to even moderate changes in dairy fat consumption $[11,19]$. Third, these two very different classes of fatty acids - the odd-chain saturated fats 15:0 and 17:0, and the natural ruminant trans-fat $t 16: \ln 7$-are intercorrelated with each other and also similarly associated with T2D. If either endogenous metabolic influences or nondairy dietary sources were a primary determinant of their levels, little plausible rationale would exist for a meaningful interrelation of these biochemically and metabolically unrelated fatty acids. Finally, while as a biomarker of dairy fat the circulating levels of these fatty acids could also be influenced by meat or fish consumption [10], such foods are not associated with lower risk of T2D in Western populations (and red meat is associated with higher risk) [33,34], so that such influences would weaken associations of these fatty acids with T2D.

A small crossover trial $(n=16)$ recently evaluated potential endogenous production of 15:0 and 17:0 from dietary fibre (inulin) and propionate (a short-chain [3-carbon] fatty acid) in comparison to cellulose $[35,36]$. The primary randomised comparison did not identify any significant effect of these factors on 15:0 or 17:0 levels. In secondary analyses evaluating pre-post

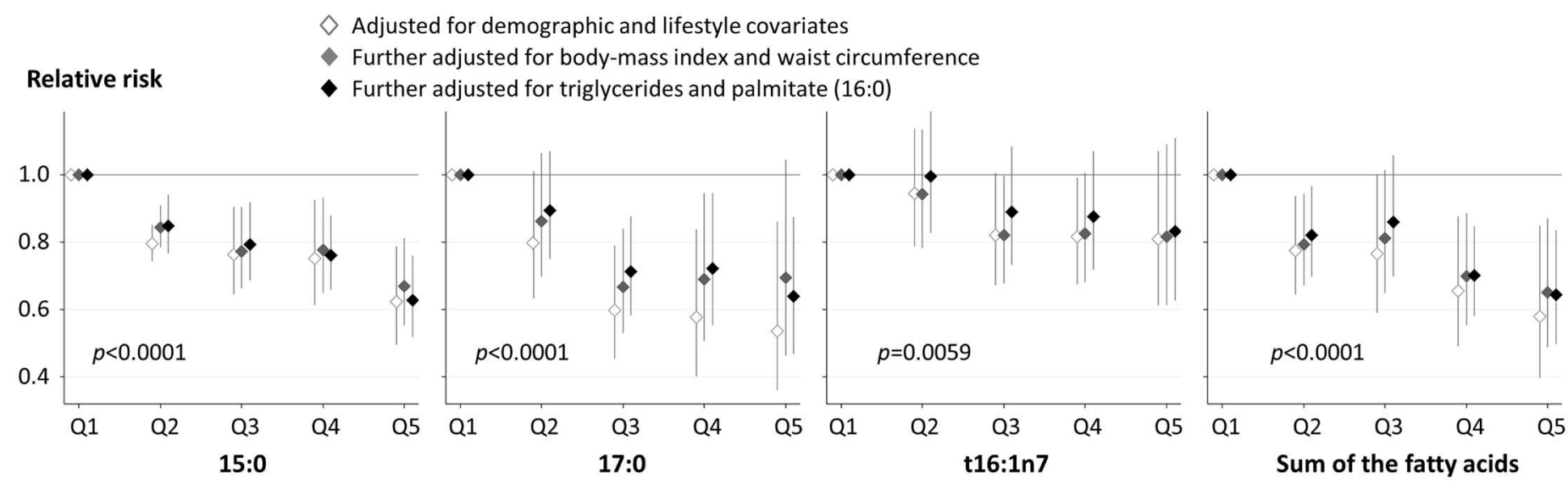

Fig 3. Prospective associations of quintile categories of fatty acid biomarkers for dairy fat consumption with the risk of developing T2D. Cohort-specific associations by quintiles were assessed in multivariable models in each cohort and pooled with inverse-variance-weighted meta-analysis. Cohort-specific multivariable adjustment was made. In the first model (open diamond), estimates were adjusted for sex, age, smoking status, alcohol consumption, socioeconomic status, physical activity, dyslipidaemia, hypertension, and menopausal status (only for women). Then, the estimates were further adjusted for BMI (grey diamond) and further adjusted for triglycerides and palmitic acid (16:0) as markers of de novo lipogenesis (black diamond). To compute $p$-values for a trend across quintiles, each fatty acid was evaluated as an ordinal variable in the most adjusted model. T2D, type 2 diabetes mellitus.

https://doi.org/10.1371/journal.pmed.1002670.g003 
(nonrandomised) levels, inulin intake was associated with higher levels of 17:0, while propionate was associated with higher 15:0 and 17:0; this was further supported by an accompanying in vitro-controlled experiment suggesting elongation of propionate into 15:0 and 17:0 using liver cancer cells [35]. The major dietary source of propionate is cheese (157 mg per $100 \mathrm{~g}$ ), in particular Swiss cheese (311 mg per $100 \mathrm{~g}$ ), with far lower levels in other dairy foods such as milk, yogurt, and cream (2-9 mg per $100 \mathrm{~g}$ ) and even lower levels in other major food groups $(<1 \mathrm{mg}$ per $100 \mathrm{~g})$ (S4 Table) [37]. High levels in cheese are plausibly related to the presence of propionate-producing bacteria and the use of sodium propionate and other propionate salts as natural preservatives and mould inhibitors in cheese [37]. These findings further support the role of odd-chain saturated fatty acids as biomarkers of dairy fat consumption, both as contained in dairy fat and as potentially synthesised from propionate in cheese [35,36].

While 15:0 has a modestly stronger correlation with self-reported dairy foods than 17:0 in some prior studies $[10,12,13,16-18]$, we found that 17:0 was more strongly associated with lower risk of T2D. Reasons for this are unclear but could reflect differences in blood lipid compartments assessed, 17:0 being a better measure of 'hidden' dairy fat in mixed foods, or possible differences in metabolic influences as mentioned above [35,38].

Our findings support the need for careful investigation to elucidate the potential biological mechanisms underlying the observed lower risk of T2D. Odd-chain fatty acids and $t 16: \ln 7$ have structural similarity to 16:0 and may interfere with lipotoxic effects of 16:0 on the pancreas [39]; it has also been hypothesised that $t 16: \ln 7$ may mimic cis-16:1n 7 and suppress hepatic de novo lipogenesis [13]. These fatty acids may also be a marker for other beneficial compounds in dairy fat or dairy-fat-rich foods such as cheese [40]. Examples of relevant constituents could include magnesium, which appears to improve hyperglycaemia and insulin resistance [41], and oestrogens, which are naturally present in dairy products $[42,43]$ and which may reduce the risk of T2D [43], as shown in two trials recruiting postmenopausal women $[44,45]$. However, these prior trials tested supradietary doses of magnesium $(>250$ $\mathrm{mg} /$ day) and oestrogens (3 $\mathrm{mg} /$ day) [41,44,45] compared with typical doses in dairy foods, approximately $20 \mathrm{mg}$ and $<0.01 \mathrm{mg}$, respectively, in $150 \mathrm{~g}$ of milk or yogurt, for example $[42,43]$. Probiotics such as in yogurt lower glucose and HbAlc in trials $[40,46]$, suggesting relevant interactions between probiotics, short-chain fatty acids, gut microbiota, and T2D [35,40]. Fermented milk and cheeses are also linked to lower risk of T2D [47], suggesting potential metabolic benefits of vitamin K2 or other compounds produced during fermentation [40]. Other constituents of dairy hypothesised to improve metabolic risk include vitamin D and calcium, but for which supplement trials do not support antidiabetic effects [48], branched-chain amino acids, but for which limited evidence suggests potential harms on insulin sensitivity [49], and animal protein, but which is not associated with lower risk of T2D [50]. Given the prevalence of dairy foods in the food supply and the prevailing conventional wisdom to avoid dairy fat, our results indicate a clear need for further clinical and biochemical investigations on $15: 0,17: 0, t 16: 1 \mathrm{n} 7$, and other components of dairy fats to clarify the mechanisms underlying our observations and help better understand roles of dairy consumption for the prevention of $\mathrm{T} 2 \mathrm{D}$ and related diseases.

In exploratory analyses, the inverse association of 15:0 and 17:0 with T2D was stronger in women than in men. Consistent with this, a meta-analysis of self-reported consumption of dairy products suggested stronger protective associations of yogurt consumption with T2D risk in women than in men $\left(\mathrm{RR}\right.$ per $50 \mathrm{~g} / \mathrm{day}=0.89$ in women and $0.97 \mathrm{in} \mathrm{men;} p_{\text {heterogeneity }}=$ 0.03) [8]. If confirmed in future studies, such an interaction may help elucidate potential mechanisms of benefit, e.g., pathways related to sex steroids [51] or starch and sugar intake (as a substitute for dairy fat) [52] and corresponding effects on atherogenic dyslipidaemia, visceral adiposity, and insulin resistance [52]. 
Our analysis has several strengths. Use of biomarkers provided measures free of limitations in self-reported dietary exposure. The similar results from several fatty acids linked to dairy fat increased confidence in the specificity of our findings. Our collaborative pooling of cohorts across different continents led to large numbers of studies, participants, and events, increasing both generalisability and statistical power. The pooling of all available cohorts minimised potential for publication bias of just the few individually significant cohorts. The standardised definitions and modelling of the populations, exposures, outcomes, and multivariable-adjusted analyses minimised bias and heterogeneity due to methodological considerations.

Potential limitations deserve consideration. The timing of diagnosis of T2D can be delayed, causing misclassification of timing in survival analysis. However, most cohorts included regular study visits and many included regular glycaemic measurements, reducing such misclassification in comparison to clinical practice. Also, any delays in diagnosis would likely be random with respect to baseline measures of fatty acid biomarkers, causing bias toward the null and increased uncertainty in estimates. Fatty acid biomarkers were assessed at baseline in each cohort, and variability over time would lead to regression dilution bias of associations toward the null. The biomarkers, despite several advantages, cannot distinguish between different food sources of dairy fat (e.g., cheese, yogurt, milk) or other foods. As an alternative to pooling of standardised participant-level analysis, all individuals could have been combined into a single dataset. Such an analysis would have a larger statistical power than our two-stage approach but require stronger assumptions, such as about covariate effects being constant across all studies [25]. Unmeasured or imprecisely measured factors may cause residual confounding, although we adjusted for major potential confounders including obesity and triglyceride levels and confirmed little difference in results across different models. Additionally, while high consumption of dairy products may be correlated with health consciousness or healthy dietary patterns in some populations [53], health-conscious consumers may have been more likely to consume low-fat than whole-fat dairy during the time periods of these studies given the prevailing dietary recommendations. Therefore residual confounding, if present, may cause underestimation of the strength of the inverse associations. As in many meta-analyses, between-study heterogeneity was evidenced and could not be fully explained. The large numbers of cases in many cohorts increased the precision of each within-study estimate, which increases the chances of finding even unimportant heterogeneity. Heterogeneity could also partly relate to varying degrees of intercorrelations between fatty acids and between tissues as well as underlying differences in populations, dietary patterns, and varieties of dairy products, including processing and fat contents. We had more limited data in nonwhite populations, requiring further research in diverse populations for which different types of dairy products may be consumed with different preparation methods.

In summary, our consortium of 16 prospective cohort studies identified significant associations of higher concentrations of 15:0, 17:0, and $t 16: \ln 7$ with lower incidence of T2D. These novel findings support the need for additional clinical and molecular research to elucidate the potential effects of these fatty acids on glucose-insulin metabolism and the potential role of selected dairy products for the prevention of T2D.

\section{Supporting information}

S1 Table. Correlations between fatty acid biomarkers for dairy fat consumption. (DOCX)

S2 Table. Correlations between fatty acid biomarkers for dairy fat consumption of two lipid fractions.

(DOCX) 
S3 Table. Prospective associations of fatty acid biomarkers for dairy fat consumption with the risk of developing T2D: Stratified analyses by regions, lipid fractions, prevalence of dyslipidaemia, and the number of fatty acids measured.

(DOCX)

S4 Table. Average amounts of naturally occurring propionate in selected foods. (DOCX)

S1 Fig. Prospective associations of fatty acid biomarkers for dairy fat consumption with the risk of developing T2D.

S2 Fig. Prospective associations of fatty acid biomarkers for dairy fat consumption with the risk of developing T2D after adjustment for adiposity measures, palmitic acid, and triglycerides.

S1 Text. Characteristics of prospective cohorts evaluating associations of fatty acid biomarkers for dairy fat consumption with the risk of developing T2D.

(DOCX)

S2 Text. Study protocol.

(DOCX)

S1 Checklist. PRISMA checklist.

(DOCX)

\section{Acknowledgments}

The authors acknowledge Eveline Waterham, Wageningen University, Wageningen, the Netherlands, for analyses of data from the Alpha Omega Cohort (AOC). Information on InterAct Consortium and FORCE can be found at http://www.inter-act.eu/ and http://force.nutrition. tufts.edu/about.

\section{Disclaimer}

This manuscript does not reflect the opinions or conclusions of any funding agency. The funders and funding organizations for participating cohorts had no role in study design, data collection, data analysis, interpretation of the data, preparation of the manuscript, and the decision to submit.

\section{Author Contributions}

Conceptualization: Fumiaki Imamura, Amanda Fretts, Liana C. del Gobbo, Luc Djoussé, Jason HY Wu, Rozenn N. Lemaitre, Dariush Mozaffarian.

Data curation: Fumiaki Imamura, Amanda Fretts, Matti Marklund, Andres V. Ardisson Korat, Catherine Helmer, Tzu-An Chen, Julie K. Bassett, Ingeborg A. Brouwer, Kuo-Liong Chien, Graham G. Giles, Vilmundur Gudnason, William S. Harris, Allison Hodge, Frank Hu, Markku Laakso, Lars Lind, Hung-Ju Lin, Ulf Risérus, Jennifer G. Robinson, Cécilia Samieri, Nona Sotoodehnia, Qi Sun, Michael Y. Tsai, Matti Uusitupa, Lynne E. Wagenknecht, Rozenn N. Lemaitre.

Formal analysis: Fumiaki Imamura, Amanda Fretts, Matti Marklund, Andres V. Ardisson Korat, Wei-Sin Yang, Maria Lankinen, Waqas Qureshi, Catherine Helmer, Tzu-An Chen, 
Kerry Wong, Julie K. Bassett, Rachel Murphy, Nathan Tintle, Chaoyu Ian Yu, Alexis C. Frazier-Wood, Barbara McKnight, Kalina Rajaobelina, Sabita S. Soedamah-Muthu.

Funding acquisition: Dariush Mozaffarian.

Investigation: Fumiaki Imamura, Amanda Fretts, Matti Marklund, Andres V. Ardisson Korat, Wei-Sin Yang, Maria Lankinen, Waqas Qureshi, Catherine Helmer, Tzu-An Chen, Kerry Wong, Julie K. Bassett, Rachel Murphy, Nathan Tintle, Chaoyu Ian Yu, Alexis C. Frazier-Wood, Liana C. del Gobbo, Johanna M. Geleijnse, Janette de Goede, Frank Hu, Albert Koulman, Barbara McKnight, David S. Siscovick, Qi Sun, Nick J. Wareham, Jason HY Wu, Renata Micha, Nita G. Forouhi, Dariush Mozaffarian.

Methodology: William S. Harris, Albert Koulman, Barbara McKnight, Dariush Mozaffarian.

Project administration: Amanda Fretts, Liana C. del Gobbo, Jason HY Wu, Renata Micha, Rozenn N. Lemaitre, Dariush Mozaffarian.

Resources: Amanda Fretts, Matti Marklund, Andres V. Ardisson Korat, Wei-Sin Yang, Maria Lankinen, Waqas Qureshi, Catherine Helmer, Tzu-An Chen, Kerry Wong, Julie K. Bassett, Rachel Murphy, Nathan Tintle, Chaoyu Ian Yu, Ingeborg A. Brouwer, Kuo-Liong Chien, Alexis C. Frazier-Wood, Luc Djoussé, Johanna M. Geleijnse, Graham G. Giles, Janette de Goede, Vilmundur Gudnason, William S. Harris, Allison Hodge, Frank Hu, Albert Koulman, Markku Laakso, Lars Lind, Hung-Ju Lin, Barbara McKnight, Kalina Rajaobelina, Ulf Risérus, Jennifer G. Robinson, Cécilia Samieri, David S. Siscovick, Sabita S. SoedamahMuthu, Nona Sotoodehnia, Qi Sun, Michael Y. Tsai, Matti Uusitupa, Lynne E. Wagenknecht, Nick J. Wareham, Nita G. Forouhi, Rozenn N. Lemaitre, Dariush Mozaffarian.

Supervision: David S. Siscovick, Dariush Mozaffarian.

Visualization: Fumiaki Imamura.

Writing - original draft: Fumiaki Imamura.

Writing - review \& editing: Fumiaki Imamura, Amanda Fretts, Matti Marklund, Andres V. Ardisson Korat, Wei-Sin Yang, Maria Lankinen, Waqas Qureshi, Catherine Helmer, TzuAn Chen, Kerry Wong, Julie K. Bassett, Rachel Murphy, Nathan Tintle, Chaoyu Ian Yu, Ingeborg A. Brouwer, Kuo-Liong Chien, Alexis C. Frazier-Wood, Liana C. del Gobbo, Luc Djoussé, Johanna M. Geleijnse, Graham G. Giles, Janette de Goede, Vilmundur Gudnason, William S. Harris, Allison Hodge, Frank Hu, Albert Koulman, Markku Laakso, Lars Lind, Hung-Ju Lin, Barbara McKnight, Kalina Rajaobelina, Ulf Risérus, Jennifer G. Robinson, Cécilia Samieri, David S. Siscovick, Sabita S. Soedamah-Muthu, Nona Sotoodehnia, Qi Sun, Michael Y. Tsai, Matti Uusitupa, Lynne E. Wagenknecht, Nick J. Wareham, Jason HY Wu, Renata Micha, Nita G. Forouhi, Rozenn N. Lemaitre, Dariush Mozaffarian.

\section{References}

1. Weaver CM. How sound is the science behind the dietary recommendations for dairy? Am J Clin Nutr. 2014; 99(5):1217S-1222S. https://doi.org/10.3945/ajcn.113.073007 PMID: 24646824

2. Lawrence GD. Dietary fats and health: dietary recommendations in the context of scientific evidence. Adv Nutr. 2013; 4(3):294-302. https://doi.org/10.3945/an.113.003657 PMID: 23674795

3. Drouin-Chartier J-P, Côté JA, Labonté M-È, Brassard D, Tessier-Grenier M, Desroches S, et al. Comprehensive Review of the Impact of Dairy Foods and Dairy Fat on Cardiometabolic Risk. Adv Nutr. 2016; 7(6):1041-51. https://doi.org/10.3945/an.115.011619 PMID: 28140322

4. Benatar JR, Sidhu K, Stewart RAH. Effects of High and Low Fat Dairy Food on Cardio-Metabolic Risk Factors: A Meta-Analysis of Randomized Studies. PLoS ONE. 2013; 8(10):e76480. https://doi.org/10. 1371/journal.pone.0076480 PMID: 24146877 
5. Abid Z, Cross AJ, Sinha R. Meat, dairy, and cancer. Am J Clin Nutr. 2014; 100 Suppl:386S-93S. https://doi.org/10.3945/ajcn.113.071597 PMID: 24847855

6. Lu W, Chen H, Niu Y, Wu H, Xia D, Wu Y. Dairy products intake and cancer mortality risk: a meta-analysis of 11 population-based cohort studies. Nutr J. 2016; 15(1):91. https://doi.org/10.1186/s12937-0160210-9 PMID: 27765039

7. Guo J, Astrup A, Lovegrove JA, Gijsbers L, Givens DI, Soedamah-Muthu SS. Milk and dairy consumption and risk of cardiovascular diseases and all-cause mortality: dose-response meta-analysis of prospective cohort studies. Eur J Epidemiol. 2017; 93(1):158-71. https://doi.org/10.1007/s10654-0170243-1 PMID: 28374228

8. Gijsbers L, Ding EL, Malik VS, de Goede J, Geleijnse JM, Soedamah-Muthu SS. Consumption of dairy foods and diabetes incidence: a dose-response meta-analysis of observational studies. Am J Clin Nutr. 2016; 103(4):1111-24. https://doi.org/10.3945/ajcn.115.123216 PMID: 26912494

9. Hebert JR, Ma Y, Clemow L, Ockene IS, Saperia G, Stanek EJ, et al. Gender Differences in Social Desirability and Social Approval Bias in Dietary Self-report. Am J Epidemiol. 1997; 146(12):1046-55. https://doi.org/10.1093/oxfordjournals.aje.a009233 PMID: 9420529

10. Allen NE, Grace PB, Ginn A, Travis RC, Roddam AW, Appleby PN, et al. Phytanic acid: measurement of plasma concentrations by gas-liquid chromatography-mass spectrometry analysis and associations with diet and other plasma fatty acids. Br J Nutr. 2008; 99(3):653-9. https://doi.org/10.1017/ S000711450782407X PMID: 17868488

11. Risérus U, Marklund M. Milk fat biomarkers and cardiometabolic disease. Current Opin Lipidol. 2017; 28(1):46-51. https://doi.org/10.1097/MOL.0000000000000381 PMID: 27906713

12. Albani V, Celis-Morales $\mathrm{C}$, Marsaux CFM, Forster H, O'Donovan CB, Woolhead C, et al. Exploring the association of dairy product intake with the fatty acids $C 15: 0$ and $C 17: 0$ measured from dried blood spots in a multipopulation cohort: Findings from the Food4Me study. Mol Nutr Food Res. 2016; 60 (4):834-45. https://doi.org/10.1002/mnfr.201500483 PMID: 26678873

13. Yakoob MY, Shi P, Willett WC, Rexrode KM, Campos H, Orav EJ, et al. Circulating Biomarkers of Dairy Fat and Risk of Incident Diabetes Mellitus Among US Men and Women in Two Large Prospective Cohorts. Circulation. 2016; 133(17):1645-54. PMID: 27006479

14. Mozaffarian D, de Oliveira Otto MC, Lemaitre RN, Fretts AM, Hotamisligil G, Tsai MY, et al. trans-Palmitoleic acid, other dairy fat biomarkers, and incident diabetes: the Multi-Ethnic Study of Atherosclerosis (MESA). Am J Clin Nutr. 2013; 97(4):854-61. https://doi.org/10.3945/ajcn.112.045468 PMID: 23407305

15. Micha R, King IB, Lemaitre RN, Rimm EB, Sacks F, Song X, et al. Food sources of individual plasma phospholipid trans fatty acid isomers: the Cardiovascular Health Study. Am J Clin Nutr. 2010; 91 (4):883-93. https://doi.org/10.3945/ajcn.2009.28877 PMID: 20219966

16. Wolk A, Furuheim M, Vessby B. Fatty acid composition of adipose tissue and serum lipids are valid biological markers of dairy fat intake in men. J Nutr. 2001; 131(3):828-33. https://doi.org/10.1093/jn/131.3. 828 PMID: 11238766

17. Brevik A, Veierød MB, Drevon CA, Andersen LF, Veierod MB, Drevon CA, et al. Evaluation of the odd fatty acids $15: 0$ and 17:0 in serum and adipose tissue as markers of intake of milk and dairy fat. Eur $J$ Clin Nutr. 2005; 59(12):1417-22. https://doi.org/10.1038/sj.ejcn.1602256 PMID: 16118654

18. Wolk A, Vessby B, Ljung $\mathrm{H}$, Barrefors $P$. Evaluation of a biological marker of dairy fat intake. Am J Clin Nutr. 1998; 68(2):291-5. https://doi.org/10.1093/ajcn/68.2.291 PMID: 9701185

19. Abdullah $\mathrm{MMH}, \mathrm{Cyr} \mathrm{A}$, Lépine $\mathrm{M}-\mathrm{C}$, Labonté $\mathrm{M}-\mathrm{E}$, Couture $\mathrm{P}$, Jones $\mathrm{PJH}$, et al. Recommended dairy product intake modulates circulating fatty acid profile in healthy adults: a multi-centre cross-over study. Br J Nutr. 2015; 113(03):435-44. https://doi.org/10.1017/S0007114514003894 PMID: 25609231

20. Golley RK, Hendrie GA. Evaluation of the Relative Concentration of Serum Fatty Acids C14:0, C15:0 and C17:0 as Markers of Children's Dairy Fat Intake. Ann Nutr Metab. 2014; 65(4):310-6. https://doi. org/10.1159/000368325 PMID: 25402168

21. Forouhi NG, Koulman A, Sharp SJ, Imamura F, Kröger J, Schulze MB, et al. Differences in the prospective association between individual plasma phospholipid saturated fatty acids and incident type 2 diabetes: the EPIC-InterAct case-cohort study. Lancet Diab Endocrinol. 2014; 2(10):810-8. https://doi.org/ 10.1016/S2213-8587(14)70146-9 PMID: 25107467

22. Mozaffarian D, Cao H, King IB, Lemaitre RN, Song X, Siscovick DS, et al. Trans-palmitoleic acid, metabolic risk factors, and new-onset diabetes in U.S. adults: a cohort study. Ann Intern Med. 2010; 153 (12):790-9. https://doi.org/10.7326/0003-4819-153-12-201012210-00005 PMID: 21173413

23. Del Gobbo LC, Imamura F, Aslibekyan S, Marklund M, Virtanen JK, Wennberg M, et al. $\omega$-3 Polyunsaturated Fatty Acid Biomarkers and Coronary Heart Disease. JAMA Intern Med. 2016; 176(8):1155. 
24. Wu JHY, Marklund M, Imamura F, Tintle N, Ardisson Korat A V, de Goede J, et al. Omega-6 fatty acid biomarkers and incident type 2 diabetes: pooled analysis of individual-level data for 39740 adults from 20 prospective cohort studies. Lancet Diabetes Endocrinol. 2017; 5(12):965-74. https://doi.org/10. 1016/S2213-8587(17)30307-8 PMID: 29032079

25. Smith-Warner SA, Spiegelman D, Ritz J, Albanes D, Beeson WL, Bernstein L, et al. Methods for pooling results of epidemiologic studies: the Pooling Project of Prospective Studies of Diet and Cancer. Am J Epidemiol. 2006; 163(11):1053-64. https://doi.org/10.1093/aje/kwj127 PMID: 16624970

26. Rosner B, Hennekens $\mathrm{CH}$, Kass EH, Miall WE. Age-specific correlation analysis of longitudinal blood pressure data. Am J Epidemiol. 1977; 106(4):306-13. https://doi.org/10.1093/oxfordjournals.aje. a112466 PMID: 910798

27. Hodge AM, English DR, O'Dea K, Sinclair AJ, Makrides M, Gibson RA, et al. Plasma phospholipid and dietary fatty acids as predictors of type 2 diabetes: interpreting the role of linoleic acid. Am J Clin Nutr. 2007; 86(1):189-97. https://doi.org/10.1093/ajcn/86.1.189 PMID: 17616780

28. Kromhout D, Giltay EJ, Geleijnse JM, Alpha Omega Trial Group. n-3 fatty acids and cardiovascular events after myocardial infarction. The New England journal of medicine. 2010; 363(21):2015-26. https://doi.org/10.1056/NEJMoa1003603 PMID: 20929341

29. Higgins JP, Thompson SG. Quantifying heterogeneity in a meta-analysis. Stat Med. 2002; 21 (11):1539-58. https://doi.org/10.1002/sim.1186 PMID: 12111919

30. Katan MB, Deslypere JP, van Birgelen AP, Penders M, Zegwaard M. Kinetics of the incorporation of dietary fatty acids into serum cholesteryl esters, erythrocyte membranes, and adipose tissue: an 18-month controlled study. J Lipid Res. 1997; 38(10):2012-22. PMID: 9374124

31. Wang DH, Jackson JR, Twining C, Rudstam LG, Zollweg-Horan E, Kraft C, et al. Saturated Branched Chain, Normal Odd-Carbon-Numbered, and n-3 (Omega-3) Polyunsaturated Fatty Acids in Freshwater Fish in the Northeastern United States. J Agric Food Chem. 2016; https://doi.org/10.1021/acs.jafc. 6b03491 PMID: 27643722

32. Ratnayake WN. Concerns about the use of $15: 0,17: 0$, and trans-16:1n-7 as biomarkers of dairy fat intake in recent observational studies that suggest beneficial effects of dairy food on incidence of diabetes and stroke. Am J Clin Nutr. 2015; 101(5):1102-3. https://doi.org/10.3945/ajcn.114.105379 PMID: 25934871

33. Pan A, Sun Q, Bernstein AM, Schulze MB, Manson JE, Willett WC, et al. Red meat consumption and risk of type 2 diabetes: 3 cohorts of US adults and an updated meta-analysis. Am J Clin Nutr. 2011; 94 (4):1088-96. https://doi.org/10.3945/ajcn.111.018978 PMID: 21831992

34. Wu JHY, Micha R, Imamura F, Pan A, Biggs ML, Ajaz O, et al. Omega-3 fatty acids and incident type 2 diabetes: a systematic review and meta-analysis. Br J Nutr. 2012; 107 Suppl:S214-27. https://doi.org/ 10.1017/S0007114512001602 PMID: 22591895

35. Weitkunat K, Schumann S, Nickel D, Hornemann S, Petzke KJ, Schulze MB, et al. Odd-chain fatty acids as a biomarker for dietary fiber intake: a novel pathway for endogenous production from propionate. Am J Clin Nutr. 2017; 105(6):1544-51. https://doi.org/10.3945/ajcn.117.152702 PMID: 28424190

36. Pfeuffer M, Jaudszus A. Pentadecanoic and Heptadecanoic Acids: Multifaceted Odd-Chain Fatty Acids. Adv Nutr. 2016; 7(4):730-4. https://doi.org/10.3945/an.115.011387 PMID: 27422507

37. EFSA Panel on Food additives and Nutrient Sources added to Food. Scientific Opinion on the re-evaluation of propionic acid ( $E 280$ ), sodium propionate (E 281), calcium propionate (E 282) and potassium propionate (E 283) as food additives. EFSA J. 2014; 12(7):3779. https://doi.org/10.2903/j.efsa.2014. 3779

38. Jenkins BJ, Seyssel K, Chiu S, Pan P-H, Lin S-Y, Stanley E, et al. Odd Chain Fatty Acids; New Insights of the Relationship Between the Gut Microbiota, Dietary Intake, Biosynthesis and Glucose Intolerance. Scientific Reports. 2017; 7:44845. https://doi.org/10.1038/srep44845 PMID: 28332596

39. Cao H, Gerhold K, Mayers JR, Wiest MM, Watkins SM, Hotamisligil GS. Identification of a lipokine, a lipid hormone linking adipose tissue to systemic metabolism. Cell. 2008; 134(6):933-44. https://doi.org/ 10.1016/j.cell.2008.07.048 PMID: 18805087

40. Mozaffarian D, Wu JHY. Flavonoids, Dairy Foods, and Cardiovascular and Metabolic Health. Circulation Res. 2018; 122(2):369-84. https://doi.org/10.1161/CIRCRESAHA.117.309008 PMID: 29348256

41. Simental-Mendía LE, Sahebkar A, Rodríguez-Morán M, Guerrero-Romero F. A systematic review and meta-analysis of randomized controlled trials on the effects of magnesium supplementation on insulin sensitivity and glucose control. Pharmacol Res. 2016; 111:272-82. https://doi.org/10.1016/j.phrs.2016. 06.019 PMID: 27329332

42. Hartmann S, Lacorn M, Steinhart $\mathrm{H}$. Natural occurrence of steroid hormones in food. Food Chemistry. 1998; 62(1):7-20. https://doi.org/10.1016/S0308-8146(97)00150-7 
43. Vicini J, Etherton T, Kris-Etherton P, Ballam J, Denham S, Staub R, et al. Survey of retail milk composition as affected by label claims regarding farm-management practices. J Am Diet Assoc. 2008; 108 (7):1198-203. https://doi.org/10.1016/j.jada.2008.04.021 PMID: 18589029

44. Margolis KL, Bonds DE, Rodabough RJ, Tinker L, Phillips LS, Allen C, et al. Effect of oestrogen plus progestin on the incidence of diabetes in postmenopausal women: results from the Women's Health Initiative Hormone Trial. Diabetologia. 2004; 47(7):1175-87. https://doi.org/10.1007/s00125-004-1448-x PMID: 15252707

45. Kanaya AM, Herrington D, Vittinghoff E, Lin F, Grady D, Bittner V, et al. Glycemic effects of postmenopausal hormone therapy: the Heart and Estrogen/progestin Replacement Study. A randomized, double-blind, placebo-controlled trial. Ann Int Med. 2003; 138(1):1-9. PMID: 12513038

46. Sun J, Buys NJ. Glucose- and glycaemic factor-lowering effects of probiotics on diabetes: a meta-analysis of randomised placebo-controlled trials. Br J Nutr. 2016; 115(07):1167-77. https://doi.org/10.1017/ S0007114516000076 PMID: 26899960

47. Sluijs I, Forouhi NG, Beulens JWJ, van der Schouw YT, Agnoli C, Arriola L, et al. The amount and type of dairy product intake and incident type 2 diabetes: results from the EPIC-InterAct Study. Am J Clin Nutr. 2012; 96(2):382-90. https://doi.org/10.3945/ajcn.111.021907 PMID: 22760573

48. de Boer IH, Tinker LF, Connelly S, Curb JD, Howard BV, Kestenbaum B, et al. Calcium plus vitamin D supplementation and the risk of incident diabetes in the Women's Health Initiative. Diabetes Care. 2008; 31(4):701-7. https://doi.org/10.2337/dc07-1829 PMID: 18235052

49. Lynch CJ, Adams SH. Branched-chain amino acids in metabolic signalling and insulin resistance. Nat Rev Endocrinol. 2014; 10(12):723-36. https://doi.org/10.1038/nrendo.2014.171 PMID: 25287287

50. Sluijs I, Beulens JWJ, van der A DL, Spijkerman AMW, Grobbee DE, van der Schouw YT. Dietary intake of total, animal, and vegetable protein and risk of type 2 diabetes in the European Prospective Investigation into Cancer and Nutrition (EPIC)-NL study. Diabetes Care. 2010; 33(1):43-8. https://doi.org/10. 2337/dc09-1321 PMID: 19825820

51. Ding EL, Song Y, Malik VS, Liu S. Sex differences of endogenous sex hormones and risk of type 2 diabetes: a systematic review and meta-analysis. JAMA. 2006; 295(11):1288-99. https://doi.org/10.1001/ jama.295.11.1288 PMID: 16537739

52. Smith JD, Hou T, Ludwig DS, Rimm EB, Willett W, Hu FB, et al. Changes in intake of protein foods, carbohydrate amount and quality, and long-term weight change: results from 3 prospective cohorts. Am J Clin Nutr. 2015; 101(6):1216-24. https://doi.org/10.3945/ajcn.114.100867 PMID: 25854882

53. Saadatian-Elahi M, Slimani N, Chajès V, Jenab M, Goudable J, Biessy C, et al. Plasma phospholipid fatty acid profiles and their association with food intakes: results from a cross-sectional study within the European Prospective Investigation into Cancer and Nutrition. Am J Clin Nutr. 2009; 89(1):331-46. https://doi.org/10.3945/ajcn.2008.26834 PMID: 19056549 